\title{
Biomechanical Modeling of the Small Intestine as Required for the Design and Operation of a Robotic Endoscope
}

\author{
H.D. Høeg, A.B. Slatkin, J.W. Burdick \\ Mechanical Engineering \\ California Institute of Technology \\ Pasadena, CA 91125
}

\author{
Dr. Warren S. Grundfest \\ Laser Research Center \\ Cedars Sinai Medical Center \\ Los Angeles, CA
}

\begin{abstract}
This paper discusses biomechanical issues that are related to the locomotion of a robotic endoscope in the human small intestine. The robot propels itself by pushing against the intestinal walls, much like a pipe crawler. However, the small intestine is not a rigid pipe; and locomotion in it is further complicated by the fact that the bowel is susceptible to damage. With the goal of engineering a safe and reliable machine, the biomechanical properties of the small bowel are studied and related to the mechanics of robotic endoscope locomotion through the small intestine.
\end{abstract}

\section{Introduction and Motivation}

This paper describes part of our ongoing effort to realize a robotic endoscope that can eventually traverse the entire human small bowel in a minimally invasive fashion. Our efforts lie in the realm of gastrointestinal endoscopy, which is the diagnosis and treatment of alimentary canal diseases by the use of flexible endoscopes. Gastroscopes provide fiberoptic or CCD camera visualization of the inner surfaces of the stomach, while colonoscopes can image the large intestine (or colon). However, these two ends represent less than $30 \%$ of the gastrointestinal tract's length, leaving the remainder (the small bowel) accessible only by invasive surgery. Estimates from the Journal of Clinical Gastroenterology indicate that small bowel diseases affect about half a million people in the United States annually. Currently, many of these patients require very invasive surgery for both diagnosis and treatment of their small bowel disease. Our envisioned robotic endoscope could provide minimally invasive access to the sections of the gastrointestinal system which are currently accessible only through invasive methods.

Ref.s $[5,6,12,13]$ more fully describe our endoscopic system concept and our preliminary efforts at developing proof-of-principle prototypes. This paper reports our efforts to understand the biomechanical issues related to robotic endoscopy in the small bowel. We need to understand these issues for the following reasons. 0-7803-5886-4/00/\$10.00@ 2000 IEEE
As part of its propulsion scheme, our proposed device laterally braces parts of its body against the compliant intestinal walls. First, we are interested to know if a robot endoscope can realize sufficient traction in a slippery and compliant intestine. More importantly, the intestine is stressed by the presence and movement of the robotic endoscope. A primary concern is to find the critical stress, or trauma threshold, beyond which irreparable (and therefore unacceptable) tissue damage occurs. Moreover, the small bowel has the following unusual feature. Due to its role in absorbing nutrients, the small bowel is one of the most highly vascularized organs in the body. The stress and strains induced in the intestinal walls by the endoscope's presence can constrict or cut off the flow of blood in the surrounding blood vessels. This stress-induced ischemia can cause temporary tissue damage that will effect the recovery of small bowel tissue after endoscopic examination, and possibly permanent damage as well.

The goal of this work is to develop quantitative analytical models (and their experimentally determined parameters) that can be used to predict the mechanical response of small bowel tissue to applied stresses and to predict the onset of ischemia. These quantitative models provide us with a means to design a machine that operates safely and reliably. After reviewing the design and function of the endoscope prototypes in Section 2, Section 3 reviews an analytical/experimental model of small bowel tissue mechanics. Section 4 then discusses the clinical aspects of ischemia and presents a quantitative model for predicting the onset of ischemia.

Relation to previous work. As described below, our design is reminiscent of "pipe crawling" robots. Fukuda et. al have developed self-propelled robotic systems for inspecting small and medium size pipes [2], and patents for these devices (such as Ref. [11]) have been granted. While the human intestine is analogous to a pipe, there are many significant differences which prevent a simple adaptation of prior pipe crawling principles to our 
problem. The diameter of the human intestine can vary by a factor of four over its length. Conventional pipe crawlers do not handle such variations. In addition, the intestine is highly flexible, fragile, and slippery. Thus, the traction mechanisms used in many prior pipe crawling devices would likely cause significant injury to the intestinal lining (provided they could produce sufficient traction at all). These significant differences motivate and necessitate the work described in this paper.

Others have recognized that endoscopy could be improved by the use of active articulation along the endoscope's length. For example, Sturges et. al. [15] have investigated endoscope design based on articulated bead-chain mechanisms. Fukuda et. al. have engineered actively bending catheter prototypes [1]. Ikuta et. al. have developed hyper-redundant robotic endoscope prototypes [7]. In all of these cases, the devices are advanced into the body by forces applied at their proximal ends, which are located outside of the patient. This approach inherently limits overall endoscope length and, hence, ultimate reach into the body. Our device does not require external forcing. Moreover, none of these prior works has attempted to determine the biomedical impacts of their design, as we do in this paper. Furthermore, the biomechanical models and parameters presented in this paper would be useful for analyzing how the operation of these other proposed devices would impact the intestines.

\section{System Review}

To make this paper self-contained, and to motivate the ensuing developments, this section briefly reviews the design and operation of our robotic endoscope prototypes. Our endoscope is an electromechanical analog of a segmented earthworm, and is shown conceptually in Fig. 1. Fig. 2 shows the $5^{\text {th }}$ generation prototype which has been tested in a live pig (Fig. 3).

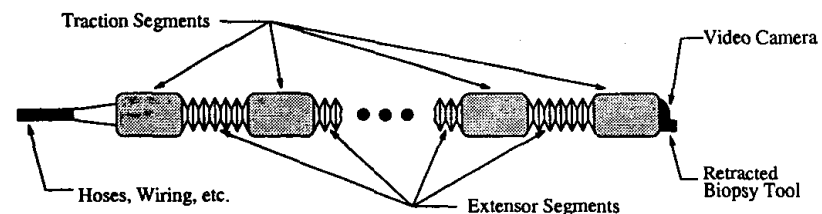

Figure 1: System schematic

This devices moves through the intestine via the action of "gripper" and "extensor" segments. The grippers are balloon-like devices whose outward radial expansion presses against the lumen wall, providing an anchoring force. The extensors, situated between the grippers, can extend or contract in length, thereby shifting the grippers and the robot's center of mass forward or backward. Locomotion can be defined as the process of generating net displacement in the bowel by

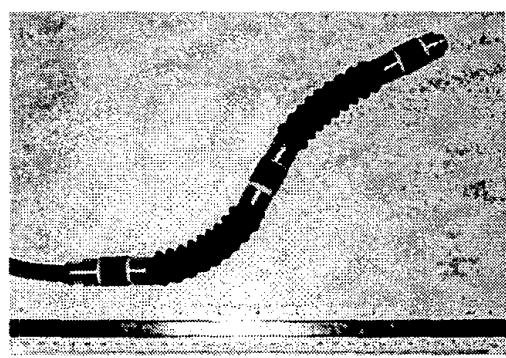

Figure 2: Generation 5 prototype

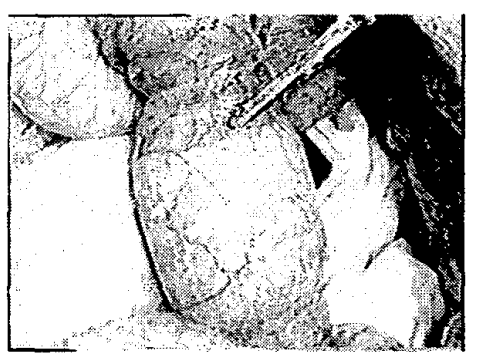

Figure 3: Segment of intestine surround inflated gripper during in vivo pig trials.

specific sequences of gripping and extension. A gait is a distinct cycle of changes in the state of the robot's gripper and extensor segments that leads to net displacement. Depending on the number of grippers and extensors, a given device can typically implement more than one gait. The simplest locomotion scheme employs two grippers and one extensor, as shown in Fig. 4. More complicated locomotion schemes involving more segments are described in Refs. $[6,5,12]$.

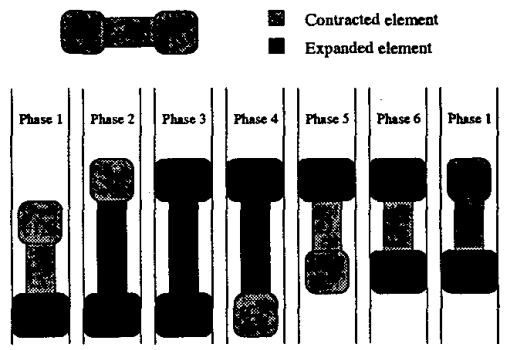

Figure 4: Schematic diagram of locomotion for a 2 gripper, 1 extensor mechanism

\section{Tissue Distension}

As the system described above moves through the small bowel, it will stress and deform the bowel tissue. This section develops an analytical and experimental model that can be used to predict the tissue behavior in response to such loading. To produce data for such a model, 3-4 inch long segments of intestine, either living or dissected, were attached between two end plugs and inflated with $\mathrm{CO}_{2}$ to a regulated internal pressure 
(Fig.s 5 and 6), as longitudinal and radial thickness measurements were taken. For live tissue measurements, the mesentary blood supply was kept in tact so that normal blood flow through the tissue prevailed.

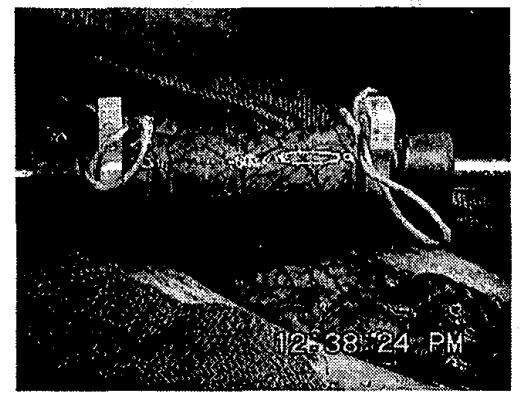

Figure 5: Low inflation pressure testing of in-vivo porcine intestine, with mesentary blood flow in tact.

The inflated state of the intestine that was produced in these experiments is not exactly the same as the loading distribution applied by the endoscope. However, our analysis below shows that excellent models for the constitutive laws of bowel tissue can be determined from this procedure. Based on these laws and further analysis, we can realize our goal of a quantitative model. The experiments included in vitro pig intestine tests, in vivo pig intestine tests, and human cadaver intestine tests. Porcine intestine was used because it most closely resembles that of humans. Live tissue responds differently to applied stresses than dead tissue. The contrast of live and dead porcine tissue studies provides a reasonably useful way to infer live human tissue response from studies of cadaver tissue.

Determining the Constitutive Relations. Our tissue specimens can be idealized as axisymmetric, homogenous, nonlinear, anistropic, viscoelastic pressure vessels undergoing large deformations due to internal axisymmetric loading distributions. The equilibrium equation equilibrium for such vessels is given by:

$$
\frac{\sigma_{m}}{\rho_{1}}+\frac{\sigma_{c}}{\rho_{2}}=\frac{p}{t}
$$

where $\sigma_{m}$ is the normal stress along a meridian, $\sigma_{c}$ is the circumferential "hoop" stress, $\rho_{1}$ is the radius of

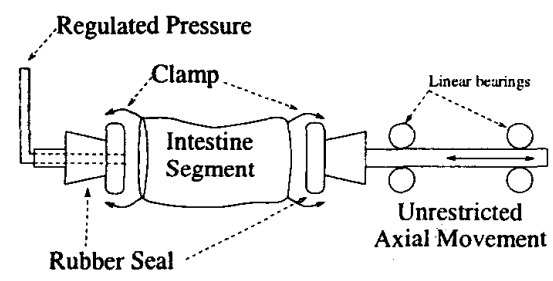

Figure 6: Test fixture schematic.

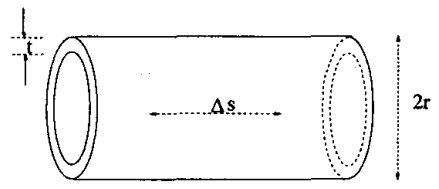

Figure 7: Simplified model for experimental specimens.

curvature of the of the meridian direction, $\rho_{2}$ is curvature normal to the meridian, $p$ is the pressure applied internally, and $t$ is the pressure vessel wall thickness.

We can use this relation to determine a number of quantities of interest. Ample evidence suggests that the intestinal tissue is essentially incompressible [9]. Let $t_{0}$, $r_{0}$, and $\Delta s_{0}$ denote the bowel segment thickess, radius, and test length ${ }^{1}$, before forces are applied (Fig. 7). The constraint of incompressibility is expressed as:

$$
\frac{t}{t_{0}}=\frac{r_{0}}{r} \frac{\Delta s_{0}}{\Delta s}
$$

For the specimen geometry seen in Fig.s 5 and 7, the meridional curvature is zero. Hence, Eq. 1 reduces to

$$
\sigma_{c}=\frac{p r}{t}=\frac{r^{2}}{t_{0} r_{0}} \frac{\Delta s}{\Delta s_{0}} p .
$$

The meridional stress in the test specimens arises from pressure forces acting on the ends of the cylinder assembly. The total force on the cylinder end is simply $T=p \pi r^{2}$, while the total tissue cross sectional area that supports this stress is $2 \pi r t$. Hence

$$
\sigma_{m}=\frac{T}{2 \pi r t}=\frac{p r}{2 t}=\frac{r^{2}}{2 t_{0} r_{0}} \frac{\Delta s}{\Delta s_{0}} p
$$

We now consider the strains in the experimental speciments induces by inflation. The meridional strain and circumferential (or "hoop") strains are given by:

$\varepsilon_{m}=\frac{\Delta s-\Delta s_{0}}{\Delta s_{0}}=\frac{\Delta s}{\Delta s_{0}}-1 ; \quad \varepsilon_{c}=\frac{2 \pi\left(r-r_{0}\right)}{2 \pi r_{0}}=\frac{r}{r_{0}}-1$

The strain in the thickness direction, assuming material incompressibility, is

$$
\varepsilon_{t}=\frac{t-t_{0}}{t_{0}}=\frac{t}{t_{0}}-1=\frac{r_{0}}{r} \frac{\Delta s_{0}}{\Delta s}-1
$$

With these equations for stress and strain, we can determine the relationship between stress and strain directly from the available experimental data of internal pressure, membrane thickness, intestinal diameter, and segment length. We generally propose nonlinear stress/strain relations of the form:

$$
\sigma_{c}=\sigma_{c}\left(\varepsilon_{c}+\nu \varepsilon_{m}+\nu \varepsilon_{t}\right) \quad \sigma_{m}=\sigma_{m}\left(\varepsilon_{m}+\nu \varepsilon_{c}+\nu \varepsilon_{t}\right)
$$




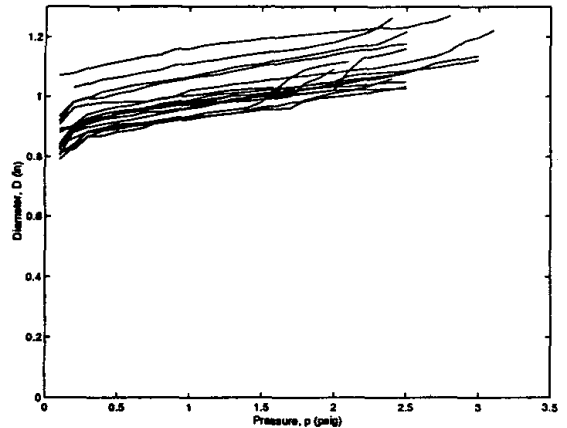

Figure 8: Trials of diameter vs. intraluminal intestinal pressure, in vivo pig

where $\nu$ is Poisson's ratio, which equals 0.5 for incompressible materials.

To obtain the data needed for the constitutive relation, numerous segments from different animals and different portions of the small bowel were inflated, while measurements of $\Delta s$ and $r$ were taken at 0.05 psi pressure increments. The nominal thickness, $t_{0}$, was measured initially, and the incompressibility constraint, Eq. 2, used to estimate $t$. Trials of Diameter vs. Internal pressure are shown in Fig. 8 for live porcine tissue. Each curve in this figure represents one trial. It is clear that the diameter can grow more than $20 \%$ under the applied pressure, suggesting that "finite deformation" elasticity theory must be used to analyze the mechanical properties. See Ref. [12] for similar results on dead porcine and human cadaver tissue.

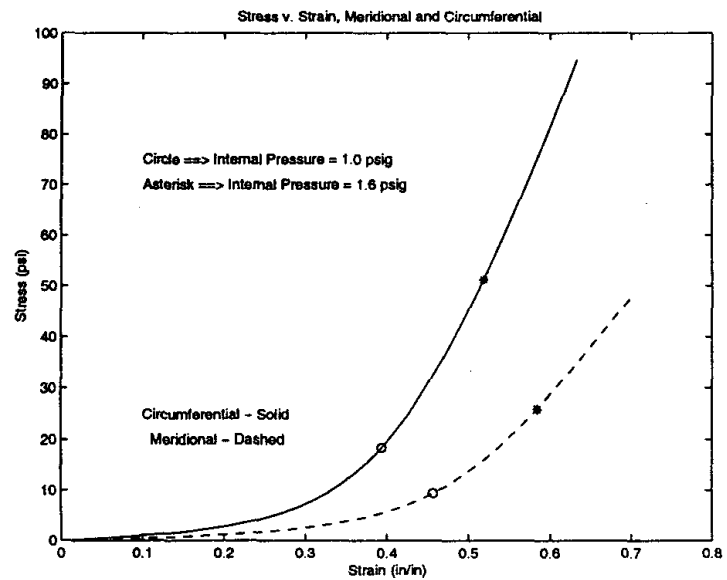

Figure 9: The anisotropic constitutive relations for invivo porcine intestine

For each trial, extrapolations of the data down to zero internal pressure were used to determine the unstressed state of the tissue segment [12]. Using this data and the stress strain relationships above, the de-

\footnotetext{
1The test length is the distance between two distinct and identified points on the intestine that are sufficiently far from the end to avoid edge effects
}

rived constitutive relations for in-vivo porcine intestine are shown in Fig. 9. Note that the tissue exhibits a nonlinear hardening characteristic, and is anisotropic, with the circumferential direction being stiffer. The asterisks on the curves indicate the stresses and strains where initial tissue tearing can be repeatedly observed.

Quantitative models and simulation. Our constitutive model forms the foundation for a computational model that simulates the response of bowel tissue to applied forces and displacements induced by a hypothetical endoscope. In general, we seek two types of output from such models. First, when tissue displacements are prescribed (such as when the gripper segment expands to a known geometry), we would like to know the resulting tissue stresses. Alternatively, when forces are applied to the tissue, we would like to compute the resulting tissue displacment field, as well as the tissue's internal stresses. For the purposes of safe endoscope design, the former problem is of greater interest, and is briefly summarized here.

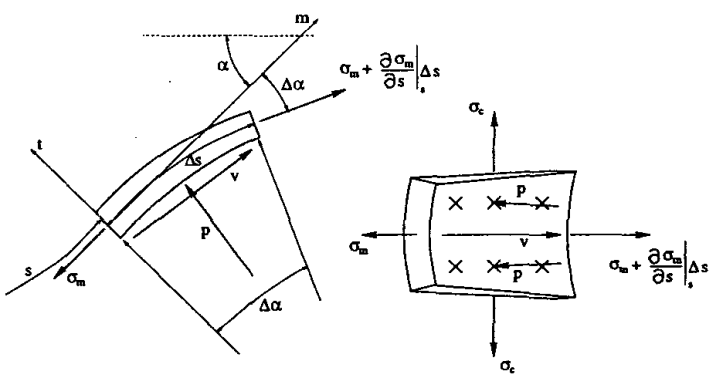

Figure 10: Free body element for derivation of the shearing traction distribution.

Additional derivations and considerations beyond the scope of this paper are needed to develop a quantitative computational model (see Ref. [12]). In particular, for the case of an imposed deformation field, the shear traction distribution must be determined. This quantity describes the surface shearing forces per unit area when the tissue contacts the endoscope in equilibrium. Using the free body diagram shown in Fig. 10 for a soft tissue element, one can derive [12] the following quasi-static expression for the shear traction distribution, $v$, which is a scalar because of the axisymmetric assumption (see Fig.10 for variable definitions):

$$
\begin{aligned}
v= & -\left[t \frac { \partial \sigma _ { m } } { \partial ( \varepsilon _ { m } + \nu \varepsilon _ { c } ) } \left[\frac{t_{0} r_{0}}{(t r)^{2}}\left(t \sin \alpha+r \frac{\partial t}{\partial s}\right)\right.\right. \\
& \left.\left.+\frac{\nu}{r_{0}} \sin \alpha\right]+\sigma_{m} \frac{\partial t}{\partial s}+\frac{\sigma_{m} t}{r} \sin \alpha\right]
\end{aligned}
$$

where the term $\frac{\partial \sigma_{m}}{\partial\left(\varepsilon_{m}+\nu \varepsilon_{c}\right)}$ is determined from the slope of the nonlinear constitutive relation in Fig. 9. Intes- 
tine is a slippery material, and hence when a displacement field is prescribed, the tissue will tend to slide along any impinging surface so as to minimize $v$. This observation is the basis for our simulation procedure.

For example, assume that a gripper has been inserted into an intestine whose unstressed diameter is given (Fig. 11). The shape of the gripper is assumed to change quasi-statically so that membrane dynamics can be ignored. This is an excellent assumption in our current prototype. As the gripper shape expands, we iteratively minimize Eq. (3) at each instant to estimate the tissue displacement along the gripper surface. With this knowledge, we can use the stress-strain relationships described above to determine the tissue stresses at each point in the material. A snapshot of the simulation is given in Fig. 11. The color coding illustrates the magnitude of the Von Mises stress in the simulated tissue. White color indicates a stress level approaching the experimentally determined stress level where tissue tearing begins. Hence, this particular gripper design and level of inflation is potentially unacceptable.

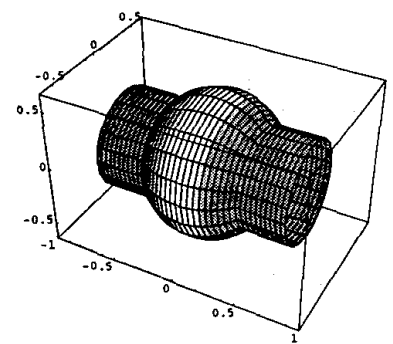

Figure 11: Simulated response of tissue to gripper expansion. The grayscale reflects the magnitude of tissue stress, where white indicates danger.

\section{Ischemia}

Blood is supplied to the small bowel through the mesentary (see Fig. 12). As the mesenteric arteries enter the wall, they branch out into arterioles (Fig. 5) which empty into a transition region where physiological exchange of gases occurs before the deoxygenated blood enters the veins [14]. When a gripper expands radially to press against the intestinal wall, the pressure in the tissue increases and eventually becomes larger than the pressure in the wall arteries. Under these conditions, the compliant arteries may partially or fully collapse. This state of ischemia can damage tissue due to blood and oxygen deprivation $[8,14]$. This section develops a model to predict the onset of ischemia due to endoscope movements. With this model, the endoscope's geometry and locomotion sequence could potentially be designed to prevent ischemia.

To develop data for the predictive model, intestinal segments were mounted as in Fig.s 5 and 6, and
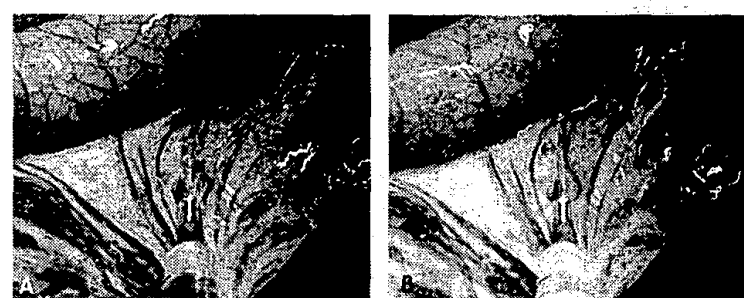

Figure 12: Backflow of blood into mesentary

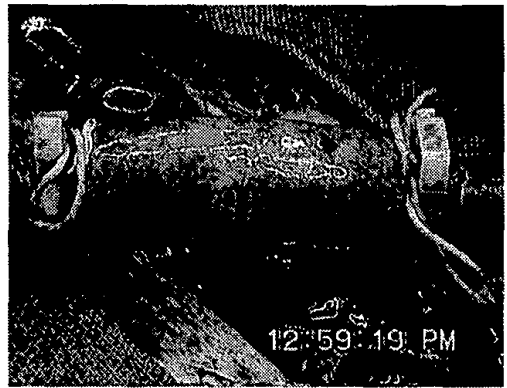

Figure 13: High inflation pressure, showing expulsion of blood

the internal pressure was gradually increased. As the pressure rose, the blood vessels became so collapsed that blood could not flow easily from the mesentary into the intestinal wall, and at a certain pressure the blood started to flow back out to the mesentary. As shown in Fig. 13, almost all of the blood had been expelled from the intestine at this pressure. While the endoscope does not generally exert forces over a large enough area to cause such massive blood flow reversal, it can induce this effect in localized regions.

To find the critical pressure that caused blood flow reversal, saline was injected (using a 26 gauge needle and $3 \mathrm{cc}$ syringe with a luer lock) at an approximately constant rate of $3 \mathrm{cc} / \mathrm{min}$ into a mesenteric artery supplying the inflated intestinal segment while gradually increasing the internal pressure and noting the pressure at which saline would no longer flow into the vessel. As the vessels collapsed with increasing inflation, saline ceased to flow into the mesentaric artery, and blood could be seen flowing into the hypodermic syringe. The measured backflow pressure was remarkably repeatable: backflow started at between 0.6 and 0.7 psi for every trial. The average was $0.67 \mathrm{psi}$, with standard deviation of 0.08 psi. This coincides very well with the values reported in Ref. [14].

\subsection{Mathematical Analysis of Ischemia}

We seek to develop a relationship between blood flow rate in the intestinal vessels and the pressure applied by the endoscope. This model should also predict the onset of ischemia. Our derivation of this model will be developed in 6 main steps: 1) develop a model for 
the pressure gradient driving the flow from the mesentery into the intestinal wall, 2) using this pressure gradient, develop an expression for the flow rate through the intestinal arteries, 3 ) couple the flow rate to arterial mechanics, 4) use constitutive relations and geometric considerations to relate intraluminal intestinal pressure to arterial deformation, 5) couple the arterial deformation to the flow rate, and 6) express arterial flow rate in terms of intraluminal intestinal pressure.

To interpret our experimental data and to develop our model, we assume the following. We reasonably assume that blood vessel tissue, intestinal tissue, and bio-fluids are incompressible. We further assume that the embedded intestinal arteries are tubes orthogonal to the intestine's longitudinal axis (Fig. 14(a)). This assumption is quite good for the main feed arteries (see Fig. 5). It is weaker, but adequate, for the branching arterioles. The inflated intestinal segments are idealized as cylinders. The inflated grippers in the current prototypes have both circumferential and meridional curvature, but as seen in Fig. 3, the inflated meridional curvature is relatively negligible, and the inflated gripper is approximately cylindrical. Note that the analysis is immediately extendable to spherical shapes.

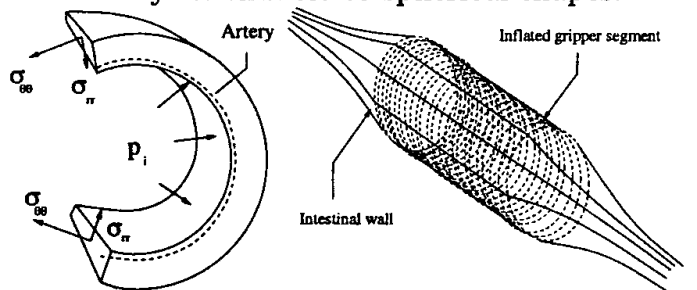

Figure 14: (a) Section of intestinal segment showing stresses and orientation of embedded artery; (b) assumed geometry of inflated gripper in intestinal lumen.

Mesenteric blood supply to the intestine. A simplified model of fluid flow in this very complex system is shown in Fig. 15(a), which depicts a single artery or arteriole embedded in intestinal tissue. This model is an adaptation of the classical "Starling resistor" model of collapsible tube flow [10]. The mesentary artery pressure, $p_{m}$, is modelled as a pressure source. The fluid perfused tissue is modelled as a chamber with a uniform regulated pressure, $p_{e}$. Thus the tube's external and exit pressures are the nominally same. In practice, this pressure is determined by the tissue stresses that arise from applied endoscope forces, and is calculated via Section 3. Hence, the pressure drop from the mesentery to the transistion region is given by:

$$
\Delta p=p_{m}-p_{e} .
$$

Intestinal arteries will generally conform to the tube law which states that the flexible tube's cross sectional area is a function of the transmural pressure. The
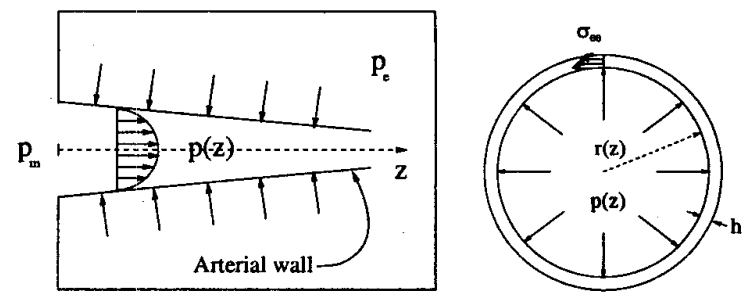

Figure 15: (a)Modified Starling Resistor model for intestinal blood supply; (b) arterial cross section.

transmural pressure is the difference between the fluid pressure inside the artery and the external pressure applied by the surrounding tissue matrix.

The tube's structural mechanics are closely coupled to its fluid mechanics: changes in fluid velocity produce pressure changes, which produce changes in cross sectional area, which in turn produce changes in the fluid velocity. Flow is restricted mainly by the reduction of the conduit's cross sectional area [3]. Generally, physiological flows are pulsatile. However, we may timeaverage the flow in the subsequent model, since pressure spikes have been largely smoothed out by frictional losses by the time the blood reaches the arterioles.

The Reynolds number for arterioles and capillaries is less than 1 (Ref. [3]), and thus the flow can be treated as laminar Poiseuille flow. This assumes that blood is a Newtonian fluid (this is not true, but it is commonly assumed in the biomechanics literature) and that the flow is fully developed (this is a valid if the grippers expand quasistatically). For such flow, the flow rate $Q$ is governed by:

$$
Q=\frac{1}{8 \mu \pi} A^{2} \frac{d p}{d z}
$$

where $\mu$ is the fluid viscosity, $A$ is the tube's cross sectional area, and $d p / d z$ is the differential pressure gradient driving the flow.

Intestinal artery length scales with the intestinal radius $R$, while variations in cross sectional area scale with $r^{2}$. Since $R \gg r$, we have two simplifying assumptions: 1) axial variation in cross sectional area is so gradual that the fluid velocity can be approximated as parallel to the $z$-axis everywhere in the flow field, and 2) the blood vessels' curvature can be ignored. Thus, we can employ Eq. (5) with the condition that the cross sectional radius is now a function of $z$.

Arterial stress and strain. We now seek relationships that govern the diameter of the artery as a function of its internal stresses and elastic properties. While the arterial constitutive relations are nonlinear, we will assume (as is often done in the literature [3]) that the constitutive relations can be described by Hooke's law. Thus, the arterial hoop and axial strain 
is given by

$$
\epsilon_{\theta \theta}=\frac{\sigma_{\theta \theta}-\nu\left(\sigma_{r r}+\sigma_{z z}\right)}{E} \quad \epsilon_{z z}=\frac{\sigma_{z z}-\nu\left(\sigma_{r r}+\sigma_{\theta \theta}\right)}{E} .
$$

For thin-walled tubes where the wall-thickness is less than $10 \%$ of the diameter, the radial stress $\sigma_{r r}$ can be neglected in comparison with the hoop stress. With this assumption, hoop stress is constant across the wall; the actual stress distribution depends on radial location and is depicted in Fig. 15(b). An analysis of the arterial cross section in Fig. 15(b) produces a simplified expression for the tube radius at an arbitrary axial location

$$
r(z)=\frac{h \sigma_{\theta \theta}(z)}{p(z)-p_{e}} .
$$

The arterial hoop strain is equal to the change in tube radius divided by the reference radius:

$$
\epsilon_{\theta \theta}=\frac{r(z)-r(0)}{r(0)} .
$$

Since the mechanical behavior of arterial and intestinal tissue is similar, an artery embedded in the intestinal wall will expand with its surrounding tissue. Therefore, the arterial axial strain is the same as the intestinal hoop strain, which is given in Section 3.

Governing flow equation. Combining the experimentally determined constitutive relations with (7) and (8), we obtain an expression for the tube radius that can be substituted into the governing flow equation (5) with boundary conditions being applied at the entry and exit region:

$$
p_{e}=p_{m}+\frac{8 \mu}{\pi} Q \int_{0}^{l} \frac{d z}{r(z)^{4}}
$$

The result of this integration is a functional relationship between the flow rate $Q$ and $p_{e}$.

Relating Luminal Distention Pressure to the Tissue Pressure. Our last step is to couple the arterial flow rate to the distention pressure exerted on the intestinal wall by the endoscope. To do this, we must find a relationship between the distention pressure, $p_{i}$, and the intestinal wall stress. A blood vessel embedded in the intestinal wall (Fig. 14) is subjected to the intestine's radial and circumferential stress (the axial stress is negligible in comparison). Hoop stresses tend to elongate the artery, while radial stresses tend to compress it laterally. Both act to decrease the vessel's cross sectional area, as accounted for by Eq. (8).

We assume that the radial stress distribution in the distended intestinal wall is linear, falling off from the intraluminal pressure $p_{i}$ at the inside walls surface to zero at the outer surface (see Ref. [16] for justification in other lumens). Since the intestinal wall's thickness is much larger than the embedded arteriole diameters [9], the compressive radial stress in the intestinal wall that acts on the tube surface is roughly constant across the vessel's diameter. With the above assumptions, the radial stress acting as compressive external pressure on an embedded vessel is approximately equal to the intraluminal intestinal pressure (see also Ref. [4])

$$
p_{e} \approx p_{i} \text {. }
$$

Strictly speaking this pressure does not act around the entire blood vessel surface. A more sophisticated model would include the possible collapsing effect of this uneven pressure distribution. However, with the elastic tube model of Fig. 15(a) the radial stress can not grow beyond the mesentery pressure before backflow of blood occurs. Therefore the tube will never be collapsed for this particular analysis.

Equation governing Ischemia. Finally, using (9) with (10), the relationship between the flow rate and the intraluminal intestinal pressure is achieved.

$$
\begin{aligned}
& Q=C\left(1-\nu f\left(p_{i}\right)\right)^{4}[ {\left[\frac{1}{\left(r_{0}\left(1-\nu^{2}\right)\left(p_{m}-p_{i}\right)-E h\right)^{3}}\right.} \\
&\left.\quad+\frac{1}{(E h)^{3}}\right] \\
& C= \frac{\pi\left(E h r_{0}\right)^{4}}{24\left(1-\nu^{2}\right) \mu r_{0} \Delta z} \\
& f\left(p_{i}\right)=a_{0} p_{i}+a_{1} p_{i}^{5}+a_{2} \arctan \left(a_{3} p_{i}\right)
\end{aligned}
$$

The parameters can be lumped into three categories: solid mechanical $(E, \nu)$, fluid mechanical $\left(\mu, p_{m}\right)$, and geometrical $\left(h, r_{0}, \Delta x\right)$. Note that artery length and fluid viscosity have no effect on how the flow rate changes with varying external tissue pressure.

The curve in Fig. 16 shows how the arterial flow rate decreases as the intestinal intraluminal pressure is increased. As the pressure is increased from 0 psig to the experimentally measured cut-off pressure of 0.7 psig, the flow rate is cut by $85 \%$, and it drops to zero as the intestinal intraluminal pressure $p_{i}$ reaches the mesentery driving pressure $p_{m}$.

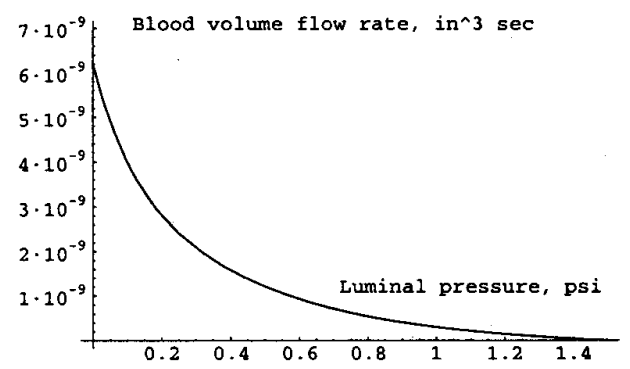

Figure 16: Prediction of change in flow rate with increasing inflation pressure. 
Parameter sensitivity analysis. A sensitivity analysis shows that in some parameter regions the model is extremely sensitive to variations in $E, p_{m}, r_{0}$, and $h$. Near the operating point $p_{i}=0.6 \mathrm{psig}$ (the experimentally measured critical pressure), the model is fairly stable with respect to appropriate ranges of $E$ and $p_{m}$ when $r_{0}$ and $h$ take very small (small capillary size) or large values (aorta size). For vessel sizes between these two extremes, the model does not as consistently exhibit the expected flow rate behavior.

Model Efficacy. The model shows reduction in blood flow as a result of endoscopic operation, as found experimentally. At the experimentally determined cut-off pressure the model predicts an $85 \%$ decrease in blood supply. This result correlates well with our experiments and with those reported in Ref. [14]. The model does not predict the complete cut-off that is observed experimentally because it does not account for arterial collapse. The model assumes a uniform pressure distribution around the tube surface. In reality the uneven distribution acts to collapse the arteries, greatly accelerating the onset of ischemia. Arterial collapse is an effect that will be considered in future models.

The current model works in conjunction with the numerical simulation presented in Section 3 which shows the stress development in the intestinal wall as it is expanded by the robot. By correlating the intraluminal pressure to the stress in the tissue, the two models can be used together to predict the critical distension value and location for the onset of ischemia.

\section{Discussion}

This paper presented analytical models and experiments that focused on some of the important biomechanical principles that must be considered in the design of a robotic endoscope that can safely self-propel itself through the small bowel. We hope to use these quantitative models to improve the design of our prototypes so that they operate within safe limits. This work should also allow other researchers to assess the impact of their proposed designs. While our simple models give good agreement with experiments, they can be improved. Future improvements will incorporate various nonlinear mechanical effects as well as models for the body's blood flow auto-regulation mechanisms. With an established upper bound on safe gripper distention, we must also determine how to produce maximal robot traction without overexpanding the intestine.

\section{References}

[1] T. Fukuda, S. Guo, K. Kosuge, F. Arai, M. Negoro, and K. Nakabayashi. Micro active catheter system with multi degrees of freedom. In IEEE Int. Conf. on Robotics and Automation, San Diego, May 1994.
[2] T. Fukuda, H. Hosokai, and M. Uemura. Rubber gas actuator driven by hydrogen storage alloy for in-pipe inspection mobile robot with flexible structure. In Proc. IEEE Int. Conf. on Robotics and Automation, pages 1847-1852, Scottsdale, AZ, 1989.

[3] Y.C. Fung. Biomechanics, Circulation, Second Edition. Springer, 1984.

[4] D.N. Granger, P.R. Kvietys, N.A. Mortillaro, and A.E. Taylor. Effect of luminal distension on intestinal transcapillary fluid exhange. American Journal of Physiology, 239:G516-G523, 1980.

[5] W.S. Grundfest, J.W. Burdick, and A.B. Slatkin. Robotic endoscopy. U.S. Patent No. 5337732, 1994.

[6] W.S. Grundfest, J.W. Burdick, and A.B. Slatkin. Robotic endoscopy continuation in part. U.S. Patent No. 5662587, 1996.

[7] K. Ikuta, M. Nokata, and S. Aritomi. Hyper-redundant active endoscope for minimum invasive surgery. In Proc. First Int. Symp. on Medical Robotics and Computer Assisted Surgery, Pittsburg, PA, 1994.

[8] L.R. Johnson, J. Christensen, M.J. Jackson, E. D. Jacobson, and J.H. Walsh. Physiology of the Gastronintestinal Tract, Vol. 1, $2^{\text {nd }}$ Ed. Raven Press, 1987.

[9] C.S. Jørgensen, F.H. Dall, S.L. Jensen, and H. Gregresen. A new combined high-frequency ultrasoundimpedance planimetry measuring system for the quantification of organ wall biomechanics in vivo. Journal of Biomechanics, 28(7):863-867, 1995.

[10] A.H. Shapiro. Steady flow in collapsible tubes. $J$. Biomechanical Engineering, 99:126-147, August 1977.

[11] Y. Shishido, H. Adachi, H. Hibino, T. Yamamoto H. Miyanaga, S. Takayama, Y. Ueda, Y. Aoki, and S. Yamaguchi. Pipe-inspecting apparatus having a self propelled unit. U.S. Patent No. 5090259, March 251986.

[12] A.B. Slatkin. Modeling and Experiments for a Class of Robotic Endoscopes. PhD thesis, California Institute of Technology, Pasadena, CA, June 1999.

[13] A.B. Slatkin and J.W. Burdick. The development of a robot endoscope. In Proc. Int. Symposium on Experimental Robotics, Stanford,CA, June 1995.

[14] American Physiological Society. Handbook of Physiology, Section 6: The Gastrointestinal System, Vol. 1 Motility and Circulation, Part 2. Oxford University Press, 1989.

[15] R.H. Sturges and S. Laowattana. A flexible, tendoncontrolled device for endoscopy. In Proc. IEEE Int. Conf. on Robotics and Automation, Sacramento, CA, 1991.

[16] K. Takamizawa and K. Hayashi. Strain energy density function and uniform strain hypothesis for arterial mechanics. Journal of Biomechanics, 20(1):7-17, 1987. 